\title{
Philosophiques
}

\section{Révolution et spéculation chez le jeune Marx}

\section{Laurent-Paul Luc}

Volume 10, numéro 2, octobre 1983

Le marxisme cent ans après Marx

URI : https://id.erudit.org/iderudit/203226ar

DOI : https://doi.org/10.7202/203226ar

Aller au sommaire du numéro

Éditeur(s)

Société de philosophie du Québec

ISSN

0316-2923 (imprimé)

1492-1391 (numérique)

Découvrir la revue

Citer cet article

Luc, L.-P. (1983). Révolution et spéculation chez le jeune Marx. Philosophiques, 10(2), 205-220. https://doi.org/10.7202/203226ar

\section{Résumé de l'article}

Énoncée comme " impératif catégorique » de l'acte d'appropriation ordonné à l'avènement de l'« Homme total ", la révolution prolétarienne dérive dans les écrits du jeune Marx d'un choix éthique s'alignant sur une ontologie de la vie moulée dans la pensée spéculative d'origine hégélienne. d'utilisation que vous pouvez consulter en ligne.

https://apropos.erudit.org/fr/usagers/politique-dutilisation/ 


\title{
RÉVOLUTION ET SPÉCULATION CHEZ LE JEUNE MARX* \\ par Laurent-Paul Luc
}

\begin{abstract}
RÉSUMÉ. Énoncée comme "impératif catégorique » de l'acte d'appropriation ordonné à l'avènement de l'" Homme total ", la révolution prolétarienne dérive dans les écrits du jeune Marx d'un choix éthique s'alignant sur une ontologie de la vie moulée dans la pensée spéculative d'origine hégélienne.

ABSTRACT. In the writings of young Karl Marx, the proletarian revolution is formulated as the "categorical imperative" of the appropriation act towards the coming of "totally unalienated Man" ; and it derives from an ethical choice, based on an ontology moulded in speculative thought from hegelian origin.
\end{abstract}

Le mot-clef qui permet d'entrevoir la direction du patient travail de pensée qui se manifeste dans les écrits du jeune Marx est le mot de révolution. Comment repérer le signifié de ce mot? $\mathrm{Y}$ a-t-il un enseignement philosophique qui se dégage des analyses auxquelles il préside ? Et quels en seraient les fondements? Telles sont les questions qui constituent l'arrière-plan du débat que je voudrais vous proposer en vous soumettant une hypothèse susceptible de circonscrire l'enjeu de cette étape sinueuse de l'itinéraire marxien. Cette hypothèse pourrait se formuler dans la proposition suivante : la théorie de la révolution qui fait irruption dans les écrits du jeune Marx est indissociable d'une pensée de la vie entamée dans l'œuvre hégélien.

\footnotetext{
* Ce texte a fair l'objer d'une communication au Congrès de l'A.C.F.A.S., Université du Québec à Trois-Rivières, le 25 mai 1983. Les références aux écrits de Karl Marx seront d'abord données au texte allemand :

K. MARX. Werke (Wissenschaftliche Buchgesellschaft), Darmstadt.

I et II. Friihe Scbriften, 1962 et 1971 ;

III/1 et III/2. Politische Schriften, 1960.

IV, V et VI. Oekonomische Schriften, 1962, 1963 et 1964.

Hrsg. von H.-J. Liber.

Abréviation : (W., tome, page).
} 
Mais débattre une telle hypothèse, n'est-ce pas risquer, comme nous en préviennent les interprétations contrastées qu'on a projetées sur une quête qui, dès 1843 , était résolue à « parvenir aux choses telles qu'elles sont, c'est-à-dire à la vérité " ${ }^{1}$ et en souci de "l'existence d'une bumanité souffrante qui pense et d'une bumanité pensante qui est opprimée " ${ }^{2}$, de s'aventurer dans une entreprise périlleuse, peut-être vaine? Qualifier de philosophique tout ce qui se cherche dans ces textes pour la plupart laissés en chantier et souvent rédigés en « rage d'ironie " ${ }^{3}$, n'estce pas, par exemple, prêter trop d'attention à ce que l'école althussérienne appelle une "bévue ${ }^{4}$ réparée dans l'Idéologie Allemande?

On sait que dans cet ouvrage destiné à confondre les JeunesHégéliens (Feuerbach, Bauer, Stirner) — « démasquer ces moutons qui se prennent et qu'on prend pour des loups " et qui " ne luttent pas le moins du monde contre le monde qui existe réellement "6-, Marx, se plaçant "d'un point de vue qui se situe en dehors de l'Allemagne " ${ }^{7}$, enracine la "présupposition ${ }^{8}$ d'une humanité historique dans "l'activité productive " d'individus déterminés, enseigne que la " transformation de l'histoire en histoire mondiale (est) une action purement matérielle, que l'on peut vérifier de manière empirique ${ }^{10}$ et soutient que pour qui « voit les choses telles qu'elles sont et se sont passées réellement, n'importe quel problème philosophique profond se résout tout bonnement en un fait empirique ${ }^{11}$. Plus rien ici ne prolonge le geste qui, dans la Dissertation, creuse l'abîme séparant un Épicure d'un Démocrite dont « les idées, estimait alors Marx, ne présentent aucun intérêt philosophique : elles ne sortent pas du cercle de la réflexion empirique ${ }^{12}$. Et

1. K. MARX, Lutber als schedsrichter zwischen Strauss und Feuerbach, W., I, 109.

2. Lettre de K. Marx à A. Ruge, mai 1843, in MARX-ENGELS, Correspondance, tome 1 (1835-1848), Paris, Éd. Sociales, 1971, p. 296, Citée : Cor., p. . . .

3. Lettre de K. Marx à son père, novembre 1837, in Cor., p. 37.

4. L. ALTHUSSER, Lire le Capital, Paris, Maspero, 1968, p. 23.

5. K. MARX/F. ENGELS, Die Deutsche Ideologie, W., II ; tr. fr. : L'Idéologie Allemande, Paris, Éd. Sociales, 1976. Abrévs. : D.I. et I.A. D.I., W., II, 9 ; I.A., p. 9.

6. D.I.,W.. II, $15 ;$ I.A., p. 15.

7. D.I. W.. II, $13 ;$ I.A., p. 13.

8. D.L., W.. II, $16 ;$ I.A., p. 15.

9. D.I., W., II, $21 ; I . A .$, p. 19.

10. D.I., W., II. 42, $43 ;$ I.A., p. 35 .

11. D.I. W.. II. 26 ; I.A., p. 24,25 .

12. K. MARX, Die Doktordissertation : Differenz der demokrttzschen und epikureischen Naturpht- 
à manier les consignes méthodiques qu'enchaîneront les œuvres de maturité pour élargir ce cercle, ne risquerait-on pas de laisser notre hypothèse à ce mauvais sort que la Sainte Famille jette à la philosophie : "Il semble (. . .) que la philosophie, précisément parce qu'elle n'était que l'expression transcendante et abstraite de l'état de choses existant, à cause de sa transcendance et de son abstraction, à cause de sa distinction imaginaire d'avec le monde, devait se figurer avoir laissé bien au-dessous d'elle l'état de choses existant et les hommes réels ; que d'autre part, parce qu'elle ne se distinguait pas réllement du monde, elle se trouvait dans l'impossibilité de porter sur lui un jugement réel, de faire jouer à son encontre un pouvoir de discernement effectif, donc d'intervenir pratiquement, mais devait se contenter tout au plus d'une pratique in abstracto. La philosophie n'était superpratique qu'en un sens : elle planait au-dessus de la pratique $" 13$.

De tels propos déjouent par avance toute tentative de déceler dans les questions que le jeune Marx se pose sur l'émancipation humaine, sur l'État et la révolution, sur les lieux et voies d'une « science effective » (wirkliche Wissenschaft $)^{14}$ libérée des simulacres que les philosophes idéologues substituent à la « vie effective » (wirklich Leben) ${ }^{15}$ sans y séjourner jamais, les signes d'une obédience inconditionnelle à l'égard de Hegel. Il ne serait sans doute pas erroné de reconnaître que la lecture de Hegel ait accompagné l'éveil de ces questions. Mais comment admettre qu'elle a contribué à en aménager le site propre ? Un voile jeté, après le meurtre rituel, sur la nudité du Père ? Ou encore, pour reprendre l'expression d'une lettre que Marx reçut de Ruge, l'inhabileté d'un "nouveau philosophe ${ }^{16}$ réduit, comme un apprenti, à penser par opposition, à emmêler catégories économiques et notions feuerbachiennes, à confondre Smith et

losopbie, W., I ; tr. fr. : Différence de la philosophie de la nature chez Démocrite et Épucure, Paris, Éd. Ducros, 1970, Abrévs. : Dif., Dissertation. Dif., W., I, 60 ; Dissertation, p. 273.

13. K. MARX/F. ENGELS, Die Heilige Famulie, oder Kritik der kritischen Kritik. Gegen Bruno Bauer une Consorten, W., I ; tr. fr. : La Sainte Famille, Paris, Éd. Sociales, 1972. Abrévs. H.F. et S.F.H.F., W., I, $709 ;$ S.F., p. 51.

14. D.I., W., II, $24 ;$ I.A., p. 21.

15. D.I., W., II, $24 ;$ I.A., p. 21.

16. Lettre de A. Ruge à K. Marx, août 1843 in Cor., p. 296.

17: K. MARX, Oekonomisch-philosophische Manuskripte, W., I ; tr. fr. : Manuscrtts de 1844, Paris, Éd. Sociales, 1962. Abrévs. : O.P.M. et M. 44. "Engels a donc eu raison d'appeler Adam Smith le Lutber de l'économie politique ». O.P.M., W., I, $585 ;$ M. 44, p. 79,80. 
Luther $^{17}$, le Bon Dieu et le Capital ${ }^{18}$ ? La filiation hégélienne ? À ce qu'il semble, rien d'autre qu'une fièvre d'étape, ou, pour attraper le clin d'œil de Goethe sur les métamorphoses accordées aux génies, une "puberté spirituelle qu'absorbera l'âge d'homme, une fois achevée la coupe « anatomique » du présent.

Ces considérations désenchantées pourraient discréditer notre débat. Elles sont pourtant précieuses. Pour une raison. Elles indiquent que le sol sur lequel nous devons appuyer la proposition que je vous ai soumise efface toute trace d'influence et fait vaciller les sûrs repères biographiques. Il ne s'agit pas de surprendre des affinements ou de favoriser des ruptures. $\grave{A}$ un tel jeu, l'apprenti finirait bien par se précéder lui-même. Comme si, à la faveur de Dieu sait quelle prémonition, Hegel avait façonné une logique de la révolution que Marx aurait ressuscitée sous une nouvelle expression! Mais on sait bien que Hegel n'a jamais salué la révolution - pas même à Tübingen où on le surnommait déjà " le vieux ": le légendaire arbre de la liberté n'est qu'un symbole qui apaise la rumeur venue d'au-delà du Rhin. On sait aussi que le tracé phénoménologique brise l'élan révolutionnaire qu'il dessine pourtant comme "un éclair (qui) érige en une fois l'image du monde nouveau ${ }^{19}$ : un Principe, même vingt siècles après, ne devient monde qu'en mourant en Histoire. De sa belle mort. «Et à la vérité, écrit Hegel, la mort la plus plate, la plus froide, sans plus de signification que l'actede-trancher une tête de chou ou d'engloutir une gorgée d'eau ${ }^{20}$.

D'où, peut-être, ce pessimisme modéré du travail philosophique pratiqué dans la Philosophie du Droit que le jeune Marx a lue à la loupe. Un travail qui, à force de « concevoir ce qui est (das was ist zu begreifen) " ${ }^{21}$, fléchit devant la "dureté abso-

18. ". . l'ouvrier est à l'égard du produit de son travail dans le même rapport qu'à l'égard d'un objet étranger. . . Il en va de même dans la religion. Plus l'homme met de choses en Dieu, moins il en garde pour lui-même ». O.P.M. W., I, 561, $562 ; M .44$, p. 57, 58.

19. Les références aux écrits de HEGEL seront d'abord données au texte allemand: G.W.F. HEGEL : Sämtliche Werke, Jubiläumsausgabe in 20 Bänden, h.g. v. H. GLOCKNER, Fr. Formmann Verlag, Suttgart, 1964. Abréviation : (J., tome, page). Pbänomenologie des Geistes, J., 2 ; tr. fr. : La Pbénoménologie de l'Esprit, Paris, Aubier, 1939, 41, 2 vol. Abrévs. : Ph.G. et Ph.E. Ph.G., J., 2, $18 ;$ Ph.E. vol. 1, p. 12. Traduction modifiée.

20. Ph.G., J., 2, 454 ; Ph.E., vol. 2, p. 136. Traduction modifiée.

21. G.W.F. HEGEL, Grundlinien der Philosopbie des Rechts, J., 7 ; tr. fr. : Principes de la philosophie du droit, Paris, Vrin, 1975. Abrévs. : Ph.R. et Pb.D. Ph.R., J., 7, 35 ; Ph.D., p. 57. Traduction modifiée. 
lue ${ }^{22}$, cède à une liberté transie de naturalité, c'est-à-dire une liberté qui ne peut être « effective " qu'en étant « besogneuse », à fleur d'arbitraire, rongée par un égoïsme si déraisonnable qu'il faut confier à la « main invisible » des institutions le " négatif » d'une volonté pourtant appelée à " être-auprès-de-soi (Bei-SichSelbst-Sein) ${ }^{23}$. Cette intimité, en voile déchiré, elle l'obtiendra le long de l'ascèse qui la conduit au-delà des libres jeux de l'art devenu avec le temps aussi insignifiant, disait Hegel, que la "tabula rasa " ${ }^{24}$, au-delà du royaume des images que fait vibrer la religion, au-delà de la "pure inquiétude de la vie ${ }^{25}$, dans la philosophie qui, "lassée des agitations suscitées par les passions immédiates dans la réalité (. . .) s'en dégage pour se livrer à la contemplation ${ }^{26}$. Mais, pour assurer si paisiblement sa mise tout en étant hors du coup, le visionnaire de l'Absolu qui, après s'être jeté « à corps perdu ${ }^{27}$ dans la philosophie, en a sonné le coup crépusculaire, a-t-il bien " pensé la pure vie (reines Leben $z u$ denken) " ${ }^{28}$ que la bataille d'Iéna a célébrée comme la lumière et la nuit du " pays d'origine de la vérité " ${ }^{29}$ ? Ce qu'il a pour son dire ne serait-il pas affecté d'une défaillance de la vue?

Ces questions traversent toute l'œúuvre de Marx. Cómmé si, obsédé par elles, il s'appliquait à ajuster un regard porté sur ce qui s'appelle à l'étourdie leś « faits" » de la vie. Pour en faire un dire qui soit un voir? Mais comment entendre cette question ? Ne risque-t-on pas, à la soulever, d'assombrir notre hypo-

22. Ph.R., J., 7, 275 ; Ph.D., p. 223. Traduction modifiée.

23. G.W.F. HEGEL, Vorlesungen über die Philosophie der Gesthichte, J., 11 ; tr. fr. : Legons sur la philosophie de l'histoire, Paris, Vrin, 1963. Abrévs. : V.P.G. et L.Ph.H. V.P.G..J., 11, 44, L.Ph.H., p. 44. Traduction modifiée.

24. G.W.F. HEGEL, Vorlesungen uiber dee Aesthetik, J., 13, 232 ; tr. fr. : Esthéttque, Paris, Aubier, 1944, vol. 5, p. 146.

25. Ph.G., J., 2, $44 ;$ Pb.E., vol. 1, p. 40.

26. V.P.G., J.; II, $569 ;$ L.Ph.H., 409.

27. G.W.F. HEGEL, Differenzdes Fichte'schen und Schelling'schen Systems der Pbilosophie, J., 1, 43 ; tr. fr. Différence des systèmes philosophiques de Fichte et de Schelling in Première publications, Paris, Ophirys, 1964, p. 85.

28. F.W.F. HEGEL, Der Geist der Christentums und sein Schicksal in Hegels theologische Jugendschriften, hg. v. H. NOHL, reproduction photomécanique par Minerva, Frankfurt, 1966, p. 302 ; tr. fr. : L'Esprit du christianisme et son destin, Paris, Vrin, 1948, p. 76.

29. G.W.F. HEGEL, Glauben und Wissen, J., 1, 432 ; tr. fr. : Foi et Savoir, in Premières publications, Paris, Ophrys, 1964, p. 298. 
thèse d'un malentendu, de ce que Habermas, par exemple, appelle " le malentendu d'une interprétation ontologique du marxisme " - s'il est vrai, comme il le proclame, que Marx « ne s'est jamais interrogé sur l'essence de l'homme et de la société en tant que telle " et que sa " seule et même question " n'a pas été " pourquoi l'être et pas plutôt rien » mais " pourquoi cet étant est-il ainsi et pas autrement ${ }^{30}$. Quoi qu'il en soit de l'unité du marxisme étrangement postulée dans cette sévère mise en garde, et en supposant que les questions que je viens de formuler ne soient pas que l'attente, les ruses d'un dernier mot qui les éteindrait, peut-être n'est-il pas inconvenant de les poser en nous tournant vers cette phrase des Manuscrits que Marx ne cessera d'interroger : "qu'est-ce que la vie sinon l'activité " 31 .

Cette question, Marx la portait en lui depuis longtemps. Ouvrons cette lettre qu'il adressa à son pêre au terme d'une année d'études à Berlin. Jetant « un regard en arrière sur les circonstances qui l'ont marquée ", l'étudiant de 19 ans détaille à son père bilan et projet de ses recherches « du même œil, précise$\mathrm{t}$-il, dont je considère la vie en général, à savoir comme l'expression d'une activité de l'esprit, qui prend ensuite corps dans toutes les directions, dans le savoir, l'art et la vie privée ${ }^{32}$. Ne cherchons pas ici l'intuition que l'œuvre à venir n'aurait eu qu'à monnayer, une intuition qui, n'étant sollicitée par aucun dehors, arc-boutée sur elle-même, s'apparenterait à celle qui, à en croire Bergson, quelque fût le temps de "Spinoza vivant et écrivant ", nous aurait assuré "d'avoir le spinozisme tout de même ${ }^{33}$.

L'effort déployé dans la Dissertation pour résoudre « un problème, insoluble jusqu'ici, de l'histoire de la philosophie grecque ${ }^{34}$, les luttes menées dans la Gazette Rbénane au nom de « la foule pauvre et dépouillée politiquement et socialement ${ }^{35}$ (dont Marx dit assez curieusement qu' " elle éprouve

30. J. HABERMAS, Theorie und Praxis, Neuwied und Berlin, Luchterhand, 1963, p. 169, 311 ; tr. fr. : Théorie et Pratique, Paris, Payot, 1975, T. II, p. 16, 209.

31. O.P.M., W., I, 565;M. 44, p. 61.

32. Lettre de K. Marx à son père, novembre 1837, in Cor., p. 29.

33. H. BERGSON, La Pensée et le Mouvant, Paris, P.U.F., 1962, p. 124.

34. Dif., W., I, 20 ; Dissertation, p. 207."

35. K. MARX, Debatten über das Holzdiebstablsgesetz, W., I, 215. 
une tendance (Trieb) légale » $)^{36}$ pour faire craquer "l'intérêt privé (qui) se considère comme le but final du monde ${ }^{37}$, les défis lancés à Ruge (« Il s'agit de pratiquer le plus de brèches possible dans l'État chrétien et d'y introduire en fraude la raison $»)^{38}$, (« mettre le vieux monde en pleine lumière et travailler positivement à la formation du nouveau ») $)^{39}$, la dénonciation de l'égoïsme qui a miné l'action révolutionnaire américaine et française («Aucun des prétendus droits de l'homme ne dépasse donc l'homme égoïste, l'homme en tant que membre de la société civile, c'est-à-dire un individu séparé de la communauté, replié sur lui-même, uniquement préoccupé de son intérêt personnel et obéissant à son arbitraire privé $»)^{40}$ ne sont pas nés d'une humeur en mal de fusionnement avec " la vie en général », mais d'une méfiance à l'égard des institutions qui en figent le style imprévisible et d'une vigilance ouverte à " toute donnée vivante, tout immédiat, toute expérience sensible, plus généralement toute expérience réelle, dont on ne peut jamais savoir à l'avance ni d'où elle vient ni où elle va $"{ }^{41}$. S'il y a une percée qui se produit dans les écrits du jeune Marx, elle pourrait bien être celle que ces lignes viennent de signer : une expérience individuée, sans cesse relancée, rebelle au savoir. Et il n'est sans doute pas absurde de penser que le Marx de la maturité y prêta encore écoute. N'est-ce pas cette expérience sauvage qui le prévint de "formuler des recettes pour les marmites de l'avenir " 42 et l'amena à doubler d'horizon l'avènement de la " société future » lorsqu'au Congrès de Gotha il prédit que la « phase supérieure de la société communiste "s'instaurera « quand le travail sera devenu non seulement le moyen de vivre mais encore le premier besoin de la vie (das erste Lebensbedürfnis) " ${ }^{43}$ ?

Pourtant, ce n'est pas à tenter un accès aux signes fragiles d'une expérience inclôturable que s'ordonnent les travaux de

36. Ibtd., 220 .

37. Ibid., 240 .

38. Lettre de K. Marx à A. Ruge, mars 1843 in Cor., p. 290.

39. Lettre de K. Marx à A. Ruge, mai 1843 in Cor., p. 296.

40. K. MARX, Zur judenfrage, W., I, 472 ; tr. fr. : La question juive, Paris, 10/18, 1968, p. 39. Traduction modifiée. Abrévs. : $Z$.J. et $Q . J$.

41. H.F., W., I, 687 ; S.F., p. 32.

42. K. MARX, Das Kapital, W., IV, V, VI ; tr. fr. : Le Capital, Paris, Éd. Sociales, 1976, 3 vol. Abrévs. : D.K. et L.C. D.K., W., IV, XXVIII ; L.C., vol. 1, p. 19.

43. K. MARX, Kritik des Gothaer Programms, W., III/2, 1024 ; tr. fr. : Critique du Programme de Gotha in Critique des Programmes de Gotha et d'Erfurt, Paris, Éd. Sociales, 1966, p. 32. Traduction modifiée. 
Marx. Encore moins à les couvrir d'étrangeté ou à les investir de moralité ou d'utopie. Rappelons-nous la critique virulente adressée à Proudhon, au "Proudhon critique ", celui qui "se demande si le malheur est la destination morale de l'homme " 44 et ce passage de la Misère de la philosophie fustigeant ces " théoriciens utopistes qui, pour obvier aux besoins des classes opprimées, improvisent des systèmes et courent après une science régénératrice. Mais, poursuit Marx, à mesure que l'histoire marche et qu'avec elle la lutte se dessine plus nettement, ils n'ont plus besoin de chercher la science dans leur esprit, ils n'ont qu'à se rendre compte de ce qui se passe devant leurs yeux (was sich vor ibren Augen abspielt) et s'en faire l'organe. Tant qu'ils cherchent la science et ne font que des systèmes, tant qu'ils sont au début de la lutte, ils ne voient (sehen) dans la misère que la misère, sans y voir (erblicken) le côté révolutionnaire, subversif, qui renversera la société ancienne. Dès ce moment, la science produite par le mouvement historique, et s'y associant en pleine connaissance de cause, a cessé d'être doctrinaire, elle est devenue révolutionnaire ${ }^{45}$. Mais, si le monde, c'est « ce qui se passe », comment y passer " en pleine connaissance de cause », c'est-àdire en renonçant à tout "idéal sur lequel la réalité devra se régler ${ }^{46}$ et en écartant les rêves d'apaisement formés, à la manière de la Pbilosophie du Droit, dans le « faux-semblant d'un connaître réel (Schein eines wirklichen Erkennens) " 47 ? C'est autour de cette question que s'affaire le mot de révolution dans les écrits du jeune Marx.

On sait que Engels a cru que Marx l'avait abolie en devenant " homme de science " : «Quand on est " homme de science », l'on n'a pas d'idéal, on élabore des résultats scientifiques, et quand on est homme de parti en outre, on combat pour les mettre en pratique. Mais quand on a un idéal, on ne peut être homme de science, car on a un parti pris d'avance ${ }^{48}$. Mais

\footnotetext{
44. H.F..W.. I, 689 ; S.F., p. 34.

45. K. MARX, Das Elend der Philosophie, W., II, 761 ; tr. fr. : Misère de la phtlosophie, Paris, Éd. Sociales, 1968, p. 133, 134.

46. D.I., W.. II, 37 ; l.A., p. 33.

47. K. MARX, Kritik des Hegelschen Staatsrechts, W., I, 268 ; tr. fr. : Critique du droit politique bégélien, Paris, Éd. Sociales, 1975, p. 45.

48. Lettre de F. Engels à P. Lafargue, août 1884 in F. ENGELS, P. et L. LAFARGUE, Correspondance, I, I (1868-1886), Paris, Éd. Sociales, 1956, p. 235.
} 
quelle est cette science qu'il faut arracher au flot de l'Histoire et qu'il suffirait d'appliquer pour qu'advienne, à même son dire, la figure du "véritable royaume de la liberté » ${ }^{49}$ quand il s'agit « avant toutes choses (de) réveiller dans le cœur des hommes le sens que l'homme a de sa propre dignité, c'est-à-dire la liberté " et que, comme Marx n'a cessé d'y insister, « seul ce sentiment, disparu du monde réel avec les Grecs et dans les vapeurs bleutées du Ciel avec le christianisme, peut refaire de notre société une communauté où les hommes réaliseraient leurs destinées les plus hautes ${ }^{50}$ ?

Dans les écrits du jeune Marx, l'élaboration de cette question engage d'emblée la tâche de brouiller tous les partages qui ont fondé la vaine assurance des philosophes dont les fameuses Thèses sur Feuerbach nous apprennent qu'ils "n'ont fait qu' interpréter diversement le monde ${ }^{51}$. Dès la Dissertation, la philosophie était prise en "défaut (Mangel) " ${ }^{52}$ : comment pourrait-elle bâtir l'indépassable dans une " totalité concrète " alors que le cercle du "devenir-philosophique du monde qui est en même temps un devenir-monde de la philosophie " se dérobe sous la « relation-de-réflexion (Reflexionsverbältnis) 》 si chère à "l'esprit théorique devenu libre en soi-même " et est toujours en passe de se briser contre "l'énergie pratique " ${ }^{53}$ ? C'est dans ce cercle que la théorie de la révolution se fraye son chemin.

Pour en fixer le centre et tarifier la relève qui accomplira "la réalisation effective de la philosophie $"$ " .

Le texte qui s'acquitte de cette tâche audacieuse est l'admirable Introduction à la Critique du droit politique bégélien, un texte qu'on peut lire comme un essai de topologie portant sur les conditions de possibilité d'une émancipation qui permettrait à l'Allemagne d'exhiber le sens de ce qui s'est profilé dans l'His-

49. D.K., W., VI, $672 ;$ L.C., vol. III, p. 742.

50. Lettre de K. Marx à A. Ruge, mai 1843 in Cor., p. 291.

51. K. MARX, Thesen über Feuerbach, W., II, tr. fr. : Thèses sur Feuerbach in MARX/ENGELS, L'Idéologie Allemande, p. 1-4. Nous ne donnerons, comme référence, que le numéro de la thèse. Thèse, no XI.

52. Dif., W.. I, 72 ; Dissertation, p. 235.

53. Dif., W., 1, 71 ; Dissertation, p. 235. Traduction modifiée.

54. Dif., W., I, 71 ; Dissertation, p. 235. 
toire. On connaît le fameux paradoxe que cette Introduction propose à ceux qui s'avisent de surmonter l'anachronisme qui tient l'Allemagne captive des maux qui affectent les États modernes : "vous ne pouvez relever (aufheben) la philosophie sans la réaliser " ni « réaliser la philosophie sans la relever ${ }^{55}$. Ce paradoxe installe une tension dans une volonté cristallisée dans deux attitudes opposées : une attitude pratique qui consiste à « tourner le dos à la philosophie "sous prétexte que loin de prendre part à la transformation de l'Allemagne la philosophie n'en justifie que le statut quo intolérable; une attitude théorique qui lie l'émancipation de l'Allemagne à une révision du discours hégélien. Marx récuse la vanité de ces deux attitudes : "Il ne suffit pas que la pensée pousse à la réalisation, la réalité ellemême doit nécessairement pousser vers la pensée ${ }^{56}$. Mais quel est le sous-entendu qui donne ainsi d'un coup toute sa poussée pour que s'illumine "la véritable scène de toute l'histoire ${ }^{57}$ ? Pour y accéder, et "afin, écrit Marx, que l'humanité se sépare avec gaîté de son passé . . . il n'y a qu'un moyen : la praxis " ${ }^{58}$. Pour celui qui se débat avec l'héritage hégélien, ce mot n'est pas innocent. C'est qu'il s'agit, au moment même où cet héritage prétend s'inscrire dans la réalité empirique de l'Histoire, de lui faire perdre pied en le haussant jusqu'au seuil de l'inédit. Contrairement aux philosophies désuètes qui font "abstraction de l'homme réel ", cet inédit veillera au "devenir-monde de la philosophie ". Le travail philosophique est alors de bâtir son Panthéon au sein de "la mêlée " appelleront bientôt "le positif ${ }^{60}$. Que cet inédit ne s'éloigne pas dès que l'on souhaite s'y accorder, c'est bien ce que déchiffrent ces phrases qui nous sont familières : « La critique de la religion s'achève avec la doctrine selon laquelle l'homme est pour l'bomme l'être suprême, donc par l'impératif catégorique qui commande de renverser toutes les conditions au sein desquelles l'homme est un être diminué, asservi, abandonné, méprisable ${ }^{61} \ldots$

55. K. MARX, Zur Kritik der Hegelschen Rechtsphilosopbie, W. , I ; tr. fr. en annexe à : Critique du droit polittque bégélien. Abrévs. : K.H.R. et Introduction. K.H.R., W., I, 495, 496 ; Introduction, p. 204. Traduction modifiée.

56. K.H.R., W., I, 498, 499 ; Introduction, p. 206. Traduction modifiée.

57. D.l., W., II, 41 ; I.A., p. 34. Traduction modifiée.

58. K.H.R., W., I, 493, 497 ; Introduction, p. 201, 205

59. K.H.R., W., I, 491 ; Introduction, p. 200.

60. O.P.M., W., I, $658 ; M .44$, p. 143.

61. K.H.R., W. , 1, 497 ; Introduction, p. 205. Traduction modifiée. 
"L'Allemagne, qui fait les choses à fond, ne peut faire-la-révolution (revolutionieren) sans faire la révolution de fond en comble. L'émancipation de l'Allemand, c'est l'émancipation de l'bomme " 22.

On pourrait s'étonner ici des accents moralistes peut-être même bibliques qu'éveille la formule empruntée à Kant, d'autant plus que dans ce texte Marx enseigne, mais aucunement pour des motifs reliés à une analyse de l'Histoire, que la libération de l'Allemagne doit être créée par la «formation (Bildung) »d'une classe, le prolétariat, alors conçue comme l'« arme » de la philosophie et le « cœur » de l' "émancipation universelle de l'homme ${ }^{63}$. Faut-il entendre dans ces accents qu'atténueront les écrits ultérieurs un écho des premiers pas que Marx vient de franchir pour s'éloigner des archipels qui risquent de surgir du sillage feuerbachien? Un «fond en comble " pour un humanisme intégral ? Mais cet optimisme radical vient de plus loin. Il est soulevé par ce que Hegel appelait " la chose même » et que Marx affronte à son tour : « Être radical, c'est saisir la chose à la racine. Or, pour l'homme, la racine, c'est l'homme lui-même " ${ }^{64}$. De quoi s'agit-il ? D'un moutonnemeni du précepte delphique qui exhausse le vœu cartésien de « marcher avec assurance en cette vie "? Il n'est pas douteux que de cette Introduction à ce que Marx appelait son "sacré livre " 65 court un fil anthropologique pour " instaurer la société juste . . . sur la base des forces productives existantes ${ }^{66}$. Une société où s'épanouit un individu que Marx présente comme un homme réel, à la fois « en chair et en os campé sur la, terre solide et bien ronde ${ }^{67}$ et "Homme total ${ }^{68}$. Un homme qui n'est pas celui qui «agit comme homme privé " ${ }^{69}$, encore moins celui qui se cherche dans les "besoins physiques" où se confine l'animal ${ }^{70}$, mais qui vit libéré du "sens de l'avoir (Sinn des

62. K.H.R..W., I, 505 ; Introduction, p. 212. Traduction modifiée.

63. K.H.R., W.. I, 503, 504, 505; Introduction, p. 211, 212.

64. K.H.R., W., I, 497 ; Introduction, p. 205. Traduction modifiée.

65. Lettre de K. Marx à F. Engels, février 1866 in Lettres sur "Le Capital ", Paris, Éd. Sociales, 1964, p. 151.

66. D.I., W., II, $242 ;$ I.A., p. 204.

67. O.P.M., W., I, $649 ; M .44$, p. 136.

68. O.P.M., W., I, $598 ; M .44$, p. 91.

69. Z.J., W., I, $461 ;$ Q.J., p. 24. Traduction modifiée.

70. ". . l'animal produit d'une façon unilatérale, tandis que l'homme produit d'une façon universelle; il ne produit que sous l'empire du besoin physique, immédiat, tandis que l'homme produit même libéré du besoin physique et ne produit vraiment que lorsqu'il en est libéré ». O.P.M., W., I, 567, 568;M. 44, p. 63, 64. 
Habens) ${ }^{71}$ et du $~ "$ monde des choses (Sachenwelt) $~^{72}$ où voudrait l'enfermer un communisme " très grossier et très irréfléchi $"{ }^{73}$, qui s'actualise dans le travail, non pas dans le « travail aliéné » que masque l'économie politique mais dans le travail dont les Manuscrits enseignent qu'il est « l'activité vitale " ${ }^{74}$ et à soi-même son " propre but (Selbstzweck) » ${ }^{75}$, travail dans lequel l'homme "se contemple (anschaut) (. . .) lui-même " ${ }^{76}$ " travail tout court (Arbeit schlechtbin) " 77 dira l'Introduction à la Critique de l'Économie politique - sans que, précise l'Idéologie Allemande, " la jouissance et le travail, la production et la consommation échoient en partage à des individus différents ${ }^{78}$.

Mais d'où ce fil anthropologique tire-t-il sa force pour que Marx puisse y faire glisser la fin de la «lutte (Kampf) » ${ }^{79}$ des forces productives, inscrire sur un même " mouvement réel $"{ }^{80}$ et sous un seul souvenir société primitive et société future, entendre dans le vacarme des machines "la grande action civilisatrice du capital " 81 ? À quelle " jouissance " Marx fait-il appel pour qu'advienne ce qu'il a en vue lorsqu'il introduit dans l'Idéologie Ällemande le mot de "métamorphose " pour souligner les lignes suivantes : " la manifestation de soi coïncide avec la vie matérielle, ce qui correspond à la transformation des individus en individus complets et au dépouillement de tout caractère imposé originairement par la nature " ${ }^{82}$ ? C'est, il me semble, dans les parages de ces questions que s'instaure ce que l'Introduction du Manuscrit de Kreusnach appelle " la doctrine » de la révolution. Explorons-les rapidement.

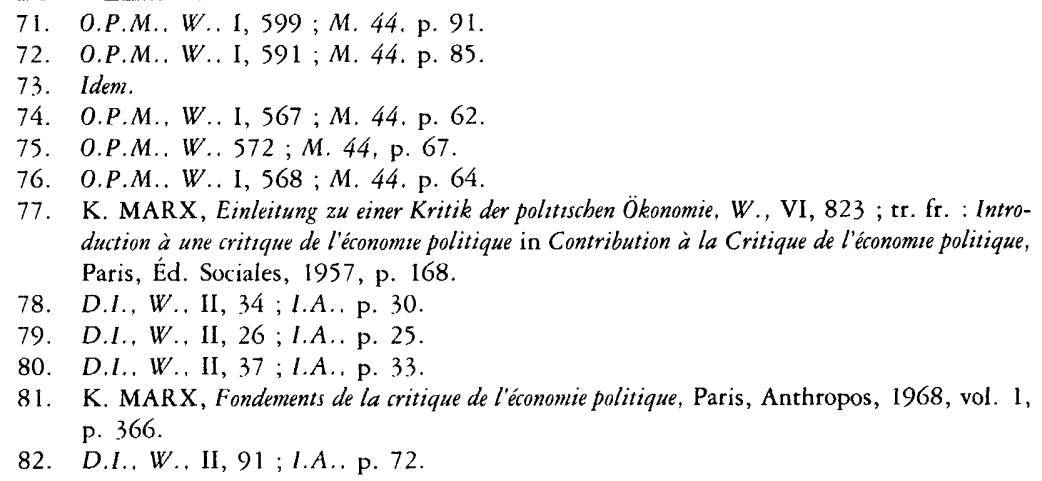


On y découvre que la nouvelle " alliance " ${ }^{83}$ que le jeune Marx veut établir entre philosophie et politique ménage, dans les interstices des choses, un accès à un « dépouillement » qui s'achève loin des lieux qu'avait patiemment fouillés la Philosophie $d u$ Droit. Car (et c'est ici que le sol de l'hypothèse que je vous ai soumise n'en est peut-être plus un) si Hegel s'effarouche lorsqu'on lui parle de révolution - sauf, bien entendu, si on lui parle en même temps de mónarchie constitutionnelle ${ }^{84}-$, s'il s'ennuie sous les nuages, s'il fait semblant de faire sa valise en ce monde, c'est qu'il n'a pas la présence d'esprit d'exorciser « ce qui se passe ». C'est cette présence d'esprit qu'allume la métamorphose marxienne par le recoupement de la nature et de la liberté. Métamorphose que Marx soustrait aux artifices de théoriciens glosant sur des codes ou s'attristant du miroitement coloré des perspectives projetées sur un labyrinthe pour la lier à une science de la vie. Écoutons-le : « là où cesse la spéculation, dans la vie effective, commence donc la science effective, positive, la présentation (Darstellung) de l'activité pratique, du processus de développement pratique des hommes. (. . .) La philosophie autonome perd avec la présentation (Darstellung) de la réalité son milieu d'existence ${ }^{85}$.

Sous le regard de cette science-là — la " remarque pure " qui tourmentait Goethe? - les figures de la vie ne sont pas celles que commémorent les sciences de la nature et les sciences de l'homme s'il ne s'y agit que de cantonner « ce qui se passe ". La tâche que s'assigne le jeune Marx est bien plutôt celle qui s'enquiert de l' " Être des hommes (Sein der Menschen) " : "L'Être des hommes est le processus de leur vie effective " ${ }^{86}$. Une quête qui, loin d'exclure la question dé la vérité, en fait, selon le vocabulaire sédimenté des Thèses sur Feuerbach, "une question pratique $"{ }^{87}$, une question de " pratique révolutionnante $"{ }^{88}$ qui

83. «Les aphorismes de Feuerbach n'ont qu'un tort à mes yeux : ils renvoient trop à la nature et trop peu à la politique. C'est pourtant la seule alliance quı peut permettre à la philosophie d'aujourd'hui de devenir vérité ". Lettre de K. Marx à A. Ruge, mars 1843 in Cor., p. 289, 290.

84. "Nous ne commençâmes à nous entendre et à nous plaire qu'en parlant de la révolution française et de la monarchie constiturionnelle ". V. COUSIN, Souvenirs d'Allemagne, in Fragments et Souvenirs, Paris, Didier, 1857.

85. D.I., W., II, $24 ;$ I.A., p. 21.

86. D.I. W.. II, $23 ; 1 . A .$, p. 20.

87. Thèse, no II.

88. Thèse, no III. 
doit mouler le «comportement authentiquement humain $" 89$. Et même une question de puissance puisqu'il s'agit de positionner de «ce côté-ci » du monde (Diesseitigkeit) ${ }^{90}$ — et pour notre temps - le centre abritant ce qui s'y manifeste : " c'est dans la pratique que l'homme a à faire la preuve de la vérité, c'est-à-dire de la réalité et de la puissance de sa pensée, la preuve qu'elle est de ce monde " ${ }^{91}$. Quelle est la vérité que le jeune Marx voit s'esquisser à même cette pratique ? Quel litige (puisqu'il s'agit de "faire la preuve ») faut-il trancher, quel concret conquérir pour qu'à la faveur de la " pratique révolutionnante " apparaisse comme dans un premier matin "l'Homme total "? On peut sonder ces questions à l'aide de l' "impératif catégorique " qui scelle l'élément révolutionnaire et sous-tend le procès que les Thèses sur Feuerbach intentent à la philosophie.

Ces Thèses tracent les grandes lignes d'une théorie universelle de l'appropriation qui disqualifie la méfiance des philosophies à l'égard du sensible : aussi bien celle du matérialisme qui ne le « saisit » pas en tant qu' « activité » que celle de l'idéalisme qui connaît bien l'activité pour en avoir développé le « côté actif » mais abstraitement, purifié de la contamination du sensible. Leur aveuglement? Fragmenter le visible sous un reflet dans un regard. D'où l'impossibilité de lui rendre justice, sauf, à la manière kantienne, à le laisser s'épeler dans la nature belle comme un « livre fermé » ou, à la manière hégélienne, à l'acheminer vers un savoir. Ce que Kant a marqué d'un $\mathrm{X}$ sans pouvoir en déceler l'équation, ce que Hegel a pris pour l'ombre de l'Esprit, Marx le fait resplendir de sa propre lumière : «On voit comment l'histoire de l'industrie et l'existence objective constituée de l'industrie sont le livre ouvert des forces humaines essentielles, la psychologie de l'homme concrètement présente, que jusqu'à présent on ne concevait pas dans sa connexion avec l'essence de l'homme, mais toujours uniquement du point de vue de quelque relation extérieure d'utilité ${ }^{92}$. Dans ce livre ouvert, les sens deviennent comme une sorte de caisse de résonnance de la nature :

89. Thèse, no I.

90. Thèse, no II.

91. Thèse, no II

92. O.P.M., W., I, 602 ; M. 44, p. 94. 
"L'œil est devenu l'œil bumain de la même façon que son objet est devenu un objet social, bumain, venant de l'homme et destiné à l'homme. Les sens sont donc devenus directement dans leur praxis des théoriciens. Ils se rapportent à la chose pour la chose, mais la chose elle-même est un rapport bumain objectif à ellemême et à l'homme et inversement. Le besoin ou la jouissance ont perdu de ce fait leur nature égoïste et la nature a perdu sa simple utilité, car l'utilité est devenue l'utilité bumaine " $" 3$. Cet enseignement-là, c'est-à-dire celui qui s'appuie sur un recoupement de la nature et de la liberté, le jeune Marx savait bien qu'il exige une métamorphose de " la conscience populaire. Le fait que la nature et l'homme sont par eux-mêmes lui est incomprébensible, parce qu'il contredit toutes les évidences de la vie pratique $"{ }^{94}$.

Ces longues citations nous rappellent que la vérité qui s'avère dans la praxis est celle qui permet à l'homme de s'assurer luimême constamment dans ses œuvres, d'en être pour ainsi dire leur effet. Même s'il doit pour un temps passer par le détour de la division du travail. Une vérité qui se pose comme un fond que ne peut surprendre aucun ailleurs, qui se recueille à travers sa propre profusion. Profusion, si vous me le permettez, qui se fusionne elle-même et même dans tout ce qui se tient autour, dans ce que Marx appelle les « circonstances " : "La coïncidence de la modification des circonstances et de l'activité humaine ou autotransformation (Selbstveränderung) ne peut être saisie et comprise rationnellement qu'en tant que pratique révolutionnante ${ }^{95}$. L'impératif de modifier les circonstances, de les transformer, de les rendre autres n'est pas celui de jouir d'un démembrement ni de prêter écoute à ce qui ne cesserait d'interpeller mais d'ouvrir un espace libre qui ferait mémoire de soi. Libre et diaphane puisque le moment du retour se déroule sur un circuit où se sont évanouies les dettes qu'avait contractées une imagination pétrie d'altérité.

On soupçonne ici la subversion que la révolution marxienne fait subir à la formule kantienne. Il ne s'agit plus de maintenir

93. O.P.M., W., I, 599 ; M. 44, p. 92.

94. O.P.M., W., I, 606 ; M. 44, p. 97.

95. Thèse, no III. 
ouverte la question de savoir qui est l'homme mais d'en fêter l'accomplissement intégral en abolissant toute distance entre ce qui est et ce qui devrait être. L'enracinement préconisé par l'émancipation de l'« homme en chair et en os, campé sur la terre solide et bien ronde " s'adresse à un homme en état de transparence totale.

Mais comment enracinement voudrait-il dire lumière si la vérité en était une de bon sable ?

Département de philosophie Université de Sherbrooke 